\title{
Inside-Out: Reflecting on your Inner State
}

\author{
Javier Hernandez \\ Daniel McDuff \\ Rich Fletcher \\ Media Lab, Massachusetts Institute of Technology \\ Cambridge, United States of America \\ \{javierhr, djmcduff, fletcher, picard $\} @$ media.mit.edu
}

Rosalind W. Picard

\begin{abstract}
We present a novel sensor system and interface that enables an individual to capture and reflect on their daily activities. The wearable system gathers both physiological responses and visual context through the use of a wearable biosensor and a cell-phone camera, respectively. Collected information is locally stored and securely transmitted to a novel digital mirror. Through interactive visualizations, this interface allows users to reflect not only on their outer appearance but also on their inner physiological responses to daily activities. Finally, we illustrate how combining a time record of physiological data with visual contextual information can improve and enhance the experience of reflection in many real-life scenarios, and serve as a useful tool for behavior science and therapy.
\end{abstract}

Keywords-component; life-logging; wearable device; electrodermal activity; digital mirror; reflection; behavior monitoring.

\section{INTRODUCTION}

Our affective state can have a large impact on our daily lives including in our decision-making, learning, work productivity and health. For more than 150 years, psychologists and behavioral scientists have thoroughly studied human emotions in laboratory settings. However, with the improvement of wearable biosensors, researchers have started to intensively study more natural and spontaneous emotions and their role in real-life interactions.

One of the major difficulties of field studies is the occurrence of unexpected and uncontrolled events that may alter or influence a person's affective state and corresponding physiology. For instance, when monitoring physiological signals such as electrodermal activity or heart rate to monitor emotional states, there are many stimuli that may result in similar autonomic responses. For instance, both stress and physical exercise may increase these signals. In order to disambiguate these cases and better understanding other confounding variables, scientists also need to unobtrusively capture contextual information. For instance, knowing that the participant is giving a talk or running on a treadmill may help differentiate between an increase associated with stress or with physical exercise.

Continuously monitoring a person's contextual activity is closely related to the concept of life-logging, where a person utilizes passive capture devices to record and digitize his life. The advancements in this area can not only help researchers to better study human behavior, but can also provide insightful information for a wide variety of applications. For instance, the monitored person can visualize his daily

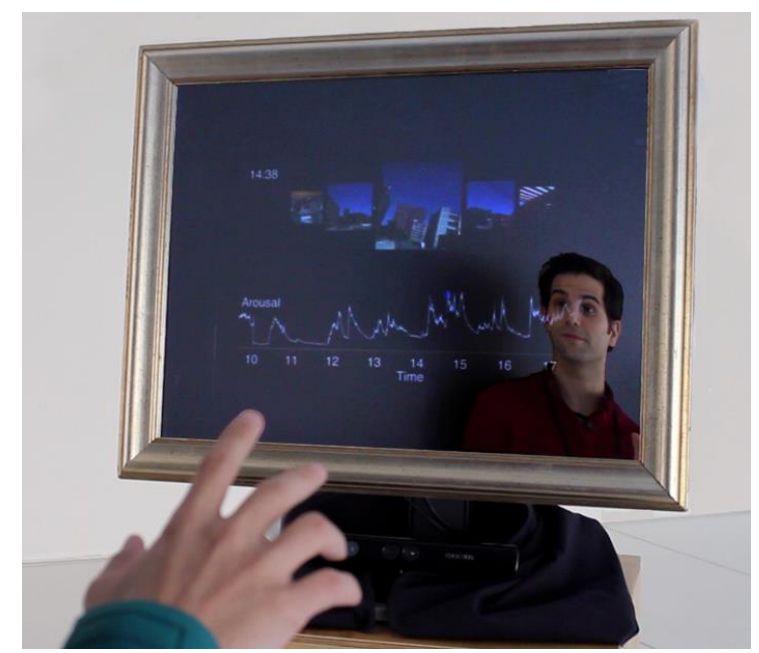

Figure 1. Inside-Out: A system for emotional reflection.

activity information to augment his memory, gain better awareness of his time allocation, or even glean insight into how certain factors may impact his health. In this work, we propose a wearable system that captures psychophysiological activation while recording contextual information, leveraging the form factors of current cellphones and biosensor technologies. Furthermore, we have created an interactive, gesturally controlled, digital mirror to display the information. This mirror allows users to naturally reflect not only on their outer appearance but also on the inner psychophysiological response. Fig. 1 shows a user interacting with the system.

\section{RELEVANT WORK}

Since the development of the first wearable systems there has been a growing interest in the field of life-logging. The seminal paper of Mann [1], envisioned the scenario in which a person uses wearable computers to capture fine grain detail of his daily activities, and uses the information to enhance his human capabilities. Since this paper was first published, there have been many approaches to efficiently capture daily-life activities. However, adequately understanding the social and ecological context of these activities has remained a key research challenge.

Among all of the contextual modalities, images are probably one of the most effective means to capture information. Visual imagery not only can convey more information than words but also appear among the preferred 
representations for autobiographical memories [2]. A successful device that passively captures images in daily-life is SenseCam [3]. Although this device was originally intended to augment human memory, it has been successfully used in other scenarios such as improving the communication of autistic individuals [4], and creating a condensed diary of daily activities [5]. SenseCam also captures other sensor modalities such as infrared light to gather additional context. However, when logging the life of a person, it is necessary to also understand how daily activities affect the inner affective state of the person.

A common method to gather the emotional responses of users involves monitoring physiological signals. For instance, Teeters et al. [6] created a system that continuously monitored the facial expressions of the camera-wearer during her daily-life activity so the person could better self-reflect on her emotional states. However, the system did not gather any additional contextual information, so the person could not accurately identify the leading factors to each emotional state. In a separate study, Ståhl et al. [7] monitored physiological information and activity of participants and asked them to manually provide contextual information through self-reports. Using the information, they created an affective diary that helped people to better reflect on their emotional responses to daily-life activities. More recently, McDuff et al. [8] created AffectAura, another affectively annotated journal for the reflection of office employees. In this case, they automatically generated the journal by analyzing several physiological signals and associating them with computer activity logs. While emotional information can potentially be associated with many other types of contextual information, few studies have used visual imagery as the context source.

One of the few exceptions and, in part motivation for this work, is the StartleCam project [9], where researchers monitored the physiological responses of computer users to detect relevant moments (e.g., startle responses) and automatically record a video with the events leading towards the physiological change. While StarleCam was ambulatory, it required a cumbersome hand-built computer with wireless module, all of which is now replaced by a cellphone. The wearable part of our project could be seen as a practical extension of StartleCam, but made with consumer hardware, continuously capturing the moments leading towards emotional responses throughout daily-life activity. Other relevant systems were proposed by Lockerd and Mueller [10], and Hori and Aizawa [11], in which they monitored physiological signals and information from other sensors to create efficient life-log retrieval systems based on context.

\section{SYSTEM}

The proposed system is comprised of two main parts: a wearable device to gather visual context and physiological data, and an interactive digital interface for users to reflect on the information.

\section{A. Wearable System}

Successful life-log capture devices become transparent and do not alter the user's behavior during daily-life activity.

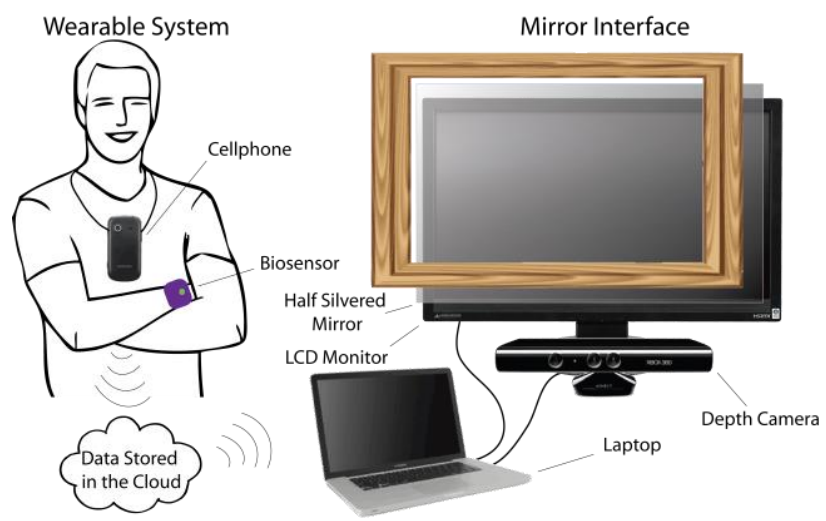

Figure 2. Schematics of the werable sensor and the digital mirror interface. The data is transferred between the two systems via the cloud.

Among other characteristics, the device should be wearable, non-intrusive, and comfortable, and should require minimal user interaction.

Fig. 2 shows the wearable sensor system. We designed a biosensor described in detail elsewhere [12] to capture physiological data. In particular, the sensor simultaneously measures electrodermal activity (EDA) and skin temperature, it also contains a 3-axis accelerometer. Since the sensor is embedded in a flexible band, it can be easily worn on several body locations (e.g., wrist, ankle). Among other benefits, the sensor is comfortable, low-cost, low-power, and wireless. Moreover it has been successfully tested in real-life settings to monitor a wide variety of populations such as soldiers with post-traumatic stress disorder and patients with drugaddiction [13].

EDA (previously known as Galvanic Skin Response) is acknowledged as one of the best indicators of sympathetic nervous system arousal, which is important in attention, emotion, and information processing, and is closely related to concepts of motivation, learning and anxiety. However, one of its main limitations is that both positive (e.g., engagement) and negative (e.g., stress) events may elicit similar responses. While measuring acceleration or skin temperature may help identify some sensor artifacts (e.g., excessive movement, loss of contact), relevant contextual information is very important in determining the valence of the responses.

Among all types of contextual information, we have chosen to gather images from the perspective of the individual. Although there are commercially available systems to retrieve visual context, we wanted to leverage the form factors of readily available technology such as current cellphones. Towards this aim, we have configured a standard mobile phone (Galaxy S Vibrant with 5 Megapixel autofocus camera) to be used as a pendant that hangs around a person's neck.

We also created a custom-made Android application to gather and synchronize the information from both the biosensor and the rear-facing camera. While the wearable biosensor transmits information over Bluetooth with a sampling rate of $4 \mathrm{~Hz}$, the cellphone camera passively captures images at a fixed sampling rate that can be adjusted 
by the user within the range of 1 to 30 images/minute. The camera system can be easily switched on and off in order to enable a privacy setting in which data should not be captured. Images are stored in JPEG format with a resolution of $150 \times 200(24 \mathrm{~KB})$, which provides enough detail to clearly represent the scene while minimizing the data volume. Additionally, if the biosensor is disconnected or is not transmitting information for any reason (e.g., discharged battery, poor Bluetooth coverage) the user is notified through cellphone vibration. As soon as the physiological and visual information is collected, the data is locally stored and securely transmitted over the Internet to the digital display.

\section{B. Mirror Interface}

One of the main benefits of real-life logging is that it enables people to reflect on and learn from their own data. With this application in mind, we designed an interactive, gesturally controlled, digital mirror interface for data reflection.

The interface consists of two main parts: a digital mirror to display information, and a gesture recognition system to browse the data. Standard mirrors are commonly used to reflect on one's outer appearance a few times a day, and the rest of time they remain unused. In this work we wanted to enhance a mirror's capabilities by incorporating the ability to display digital information, thus allowing users to reflect not only on their outer appearance but also on the inner state throughout their day. In order to allow natural interaction with the system, we installed a Kinect camera below the mirror and created custom-made gesture recognition software. The user could then navigate the presented information by waving his arm (up, down, left and right) in front of the camera.

One of the key challenges when browsing life-logged data is the vast amount of generated information. By monitoring physiological data such as EDA, which tends to be activated by personally significant events, the person can more efficiently retrieve, browse and organize the significant moments. Fig. 3 illustrates two visualizations that link the visual context with the physiological information:

1) Timeline: A person's EDA signal from the entire day is presented as a time series plot, along with a sequence of images that are syncrhonized to the EDA timeline. The user can naturally explore the data by moving her arm left/right to find earlier/later moments, and by waving her arm up/down to zoom-in/zoom-out the time series. As shown on the top visualization of Fig. 3, the blue circle indicates the moment in time the user is exploring: here it is the action of paying for food at a food truck.

2) Mosaic: The system selects images associated with the greatest electrodermal activation (amplitude of the signal) for certain periods of time (e.g., hour, day), and arranges them as a mosaic with the their sizes propotional to their activation. This visualization is especially useful as a summarization tool to quickly review emotionally significant moments. As shown in Fig. 3, the most arousing event on that particular day was positive and occurred when
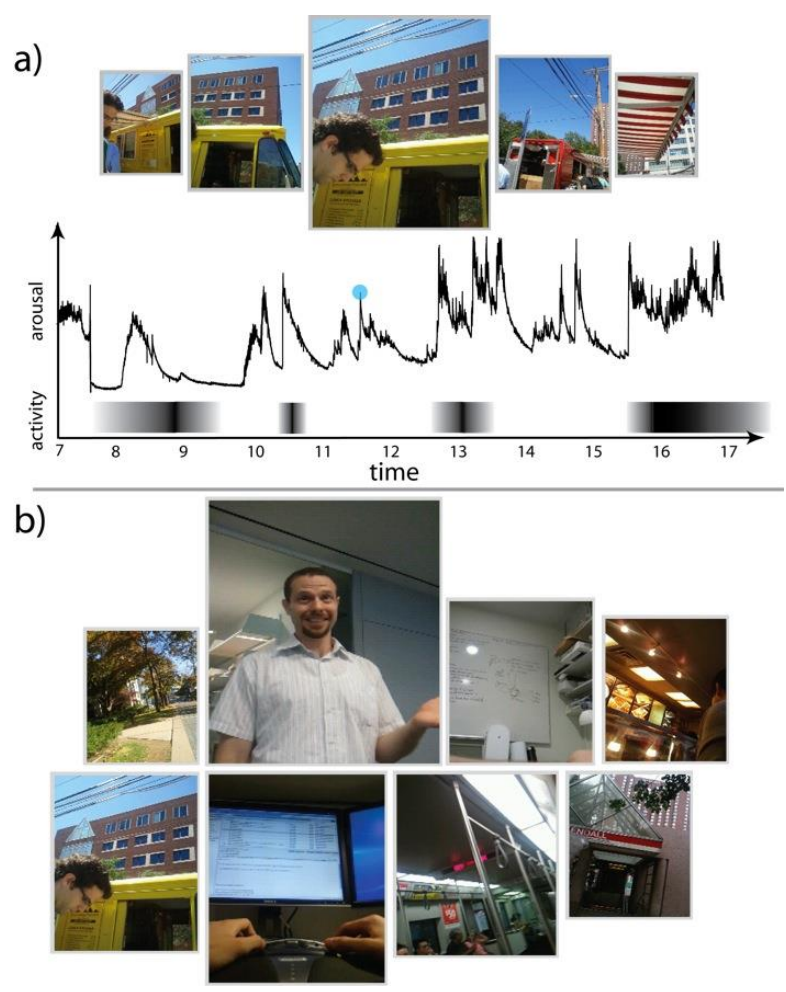

Figure 3. (a) Timeline and (b) mosaic visualizations linking physiological and visual imagery of one day.

having a funny conversation with a friend. The second most arousing event was negative and occurred when replying to a negative e-mail at the office. Finally, the least arousing event occurred when walking on a quiet street.

\section{APPLICATIONS}

Traditional research in the field of life-logging has primarily studied the effect of providing recorded information to the monitored person. For instance, if a person can review his daily activities, he can potentially augment his memory and gain more awareness of his time allocation. For these cases, physiological information is especially helpful as it has been shown to deeply influence memory acquisition and inform the decision-making processes [14]. As illustrated in this paper, physiological signals can themselves be used as an index to better retrieve and browse significant moments in time. Similarly, a person who goes on vacation could relatively quickly generate an album of photos with all the emotionally significant moments. The number of possible applications increases if the data are explored by people who were not monitored. A an example, some of the most prevalent and disruptive symptoms of Autism Spectrum Disorders (ASD) include stressful challenging behaviors (e.g., self-injury, repetitive behaviors) and impaired verbal communication. If teachers, therapists or family members could also have access to information of the internal state of people with ASD, they could potentially gain deeper understanding of the emotional states of the individuals and prevent the occurrence of 
challenging behavior. Finally, long-term physiological information would also be helpful to doctors so they can better assess the symptoms of their patients and make better diagnoses of chronic conditions (e.g., epilepsy, anxietydisorders, depression).

\section{ONGOING WORK}

The system has been tested for over 30 days by one user. The main goals of this preliminary evaluation were to ensure the system worked reliably, and to identify the main advantages and limitations.

With a sampling rate of 12 images per minute, the cellphone battery lasted for around 6 hours/day on average, which can be extended by decreasing the sampling rate. The battery of the biosensor lasted for up to 24 hours whilst recording. The number of collected images per day was around 5400, which is approximately 130 megabytes of data. The file containing the sensor data was around 5 megabytes. Therefore, with the default internal memory of the utilized cellphone (16 gigabytes) and the chosen sampling rates, the system could potentially store information of 125 days. In practice, the information is transmitted over the Internet so there is no need to keep it on the device.

The wearable sensor system was found to be comfortable and unobtrusive as all data is wirelessly transmitted (over the Internet and Bluetooth), and there was no need to carry additional devices (e.g., laptops, head-mounted cameras). Furthermore, the biosensor could be easily concealed below the clothing. One of the additional benefits is that the application runs on Android, so the system can easily be scaled to devices with different form factors (e.g., screen sizes, camera resolutions). One practical limitation of the system is the reduced field of view of cellphone cameras; however, there are commercial camera lenses that can be mounted to the cellphone to increase the field of view.

As with any image data captured in public places, privacy issues must be considered carefully. Similar privacy concerns as those observed in previous studies with SenseCam [15] were also observed throughout our evaluation. However, since the testing was primarily performed in a technical university campus, most people were willing to participate and showed interest in knowing more. Nevertheless, when moving off campus (e.g. grocery store, subway) some people acted more concerned and, in some cases, avoided being in front of the camera.

\section{CONCLUSIONS}

This work proposes a system that allows users to comfortably capture and naturally reflect on their physiological data throughout daily-life activity. In order to passively gather information, we have designed a wireless system that leverages the advancements of currently available biosensors and cellphone technology. In order to visualize the information, we augmented the capabilities of an ordinary mirror and created interactive visualizations that efficiently summarize daily activities.

In the future, we plan to perform a more thorough and methodological evaluation of both the ambulatory system and the mirror interface. Furthermore, we want to monitor several people for weeks and assess whether providing the information to the users affects their physiological awareness and impacts any behaviors.

Linking both physiological data and visual imagery in real-life settings not only may help to find more emotionally significant moments of people, but also can enhance the communication between people, catalyze introspection, inform medical diagnoses, and improve scientific understanding of psychophysiology in natural settings.

\section{ACKNOWLEDGMENTS}

This material is based upon work supported by the National Science Foundation under Grant No. NSF CCF1029585 and the MIT Media Lab Consortium.

\section{REFERENCES}

[1] S. Mann, "Smart Clothing: The Wearable Computer and WearCam," in Personal Technologies, vol. 1 (1), March 1997, pp. 21-27.

[2] W. F. Brewer, "What is Autobiographical Memory?," in D. Rubin (Ed.), Autobiographical Memory (pp. 25-49), Cambridge: Cambridge University Press, 1986.

[3] J. Gemmell, L. Williams, K. Wood, R. Lueder, and G. Bell, "Passive Capture and Ensuing Issues for a Personal Lifetime Store," in Proceedings of the Workshop on Continuous Archival and Retrieval of Personal Experiences, October 2004, pp. 48-55.

[4] G. Marcu, A. K. Dey, and S. Kiesler, "Parent-driven Use of Wearable Cameras for Autism Support: a Field Study with Families," in Proceedings of Ubiquitous Computing, September 2012, pp. 401-410.

[5] S. Hodges, L. Williams, E. Berry, S. Izadi, J. Srinivasan, A. Butler, G. Smyth, N. Kapur, and K. Wood, "SenseCam: a Retrospective Memory Aid," in Proc. of Ubiq. Comp., Sept. 2006, pp. 177-193.

[6] A. Teeters, R. Kaliouby, and R. W. Picard, "Self-Cam: Feedback from What Would Be Your Social Partner," in ACM SIGGRAPH 2006 Research Posters, August 2006.

[7] A. Ståhl, K. Höök, M. Svensson, A. Taylor, and M. Combetto, "Experiencing the Affective Diary," in Personal and Ubiquitous Computing, 13 (5), June 2009, pp. 365-378.

[8] D. McDuff, A. Karlson, A. Kapoor, A. Roseway, and M. Czerwinski, "AffectAura: an Intelligent System for Emotional Memory," in Proc. of Computer Human Interaction, May 2012, pp. 849-858.

[9] J. Healey and R. Picard, "StartleCam: A Cybernetic Wearable Camera," in Proc. of Wearable Computers, October 1998, pp. 42-49.

[10] A. Lockerd and F. Mueller, "LAFCam: Leveraging Affective Feedback Camcorder," in Comp. Hum. Int. Extended Abstracts on Human Factors in Computing Systems, April 2002, pp. 574-575.

[11] T. Hori and K. Aizawa, "Context-based Video Retrieval System for the Life-log Applications," in Proceedings of the SIGMM International Workshop on Mul. Inf. Ret., November 2003, pp. 31-38.

[12] R. Fletcher, K. Dobson, M. S. Goodwin, H. Eydgahi, O. WilderSmith, D. Fernholz, Y. Kuboyama, E. B. Hedman, M. Poh, and R. W. Picard, "iCalm: Wearable Sensor and Network Architecture for Wirelessly Communicating and Logging Autonomic Activity," in Trans. Info. Tech. Biomed., vol. 14 (2), March 2010, pp. 215-223.

[13] E. W. Boyer, R. Fletcher, R. F. Fay, D. Smelson, D. Ziedonis, and R. W. Picard, "Preliminary Efforts Directed Toward the Detection of Craving of Illicit Substances: The iHeal Project," Journal of Medical Toxicology, vol. 8 (1), March 2012, pp. 5-9.

[14] E. Peters, D. Västfjäll, T. Gärling, and P. Slovic, "Affect and Decision Making: A 'hot' topic," Journal of Behavioral Decision Making, vol. 19 (2), April 2006, pp. 79-85.

[15] D. H. Nguyen, G. Marcu, G. R. Hayes, K. N. Truong, J. Scott, M. Langheinrich, and C. Roduner, "Encountering SenseCam: Personal Recording Technologies in Everyday Life," in Proceedings of Ubiquitous Computing, September 2009, pp. 165-174. 